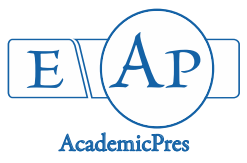

Sharma AD and Kaur I (2020)

Notulae Scientia Biologicae 12(3):536-545

DOI: $10.15835 / \mathrm{nsb} 12310711$

Research Article

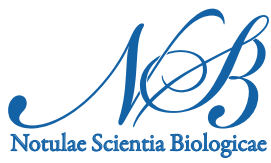

\title{
Molecular docking and pharmacokinetic screening of eucalyptol ( 1,8 cineole) from eucalyptus essential oil against SARS-CoV-2
}

\author{
Arun D. SHARMA*, Inderjeet KAUR \\ Lyallpur Khalsa College Jalandhar, PG Department of Biotechnology, India; arundevsharma47@gmail.com (*corresponding author)
}

Abstract

SARS-CoV-2 (COVID-19), member of corona virus family, is a positive single stranded RNA virus. Due to lack of drugs it is spreading its tentacles across the world. Being associated with cough, fever, and respiratory distress, this disease caused more than $15 \%$ mortality worldwide. Mpro/3CLpro has recently been regarded as a suitable target for drug design due to its vital role in virus replication. The current study focused on the inhibitory activity of eucalyptol (1,8 cineole), an essential oil component from eucalyptus oil, against $\mathrm{M}^{\mathrm{pro}} / 3 \mathrm{CL}^{\mathrm{pro}}$ from SARS-CoV-2. Till date there is no work is undertaken on in-silico analysis of this compound against $\mathrm{M}^{\mathrm{pro}} / 3 \mathrm{CL}^{\text {pro }}$ of SARS-CoV-2. Molecular docking studies were conducted by using 1-click dock tool and Patchdock analysis. In-silico absorption, distribution, metabolism, excretion and toxicity (ADMET) profile were also studied. The calculated parameters such as docking score indicated effective binding of eucalyptol to COVID-19 Mpro protein. Active site prediction revealed the involvement of active site residues in ligand binding. Interactions results indicated that, $\mathrm{M}^{\mathrm{pro}} / 3 \mathrm{CL}^{\mathrm{pro}} / \mathrm{eucalyptol}$ complexes forms hydrophobic interactions. ADMET studies provided guidelines and mechanistic scope for identification of potent anti-COVID 19 drug. Therefore, eucalyptol may represent potential herbal treatment to act as COVID-19 $\mathrm{M}^{\mathrm{pro}} / 3 \mathrm{CL}^{\mathrm{pro}}$ inhibitor, a finding which must be validated in vivo.

Keywords: COVID-19; eucalyptus; screening

\section{Introduction}

A new corona virus, 2019-n-CoV, caused a pandemic of pulmonary disease (COVID-19) in the city of Wuhan, has since spread globally (Liu and Wang, 2020). The virus has been named as SARS-CoV-2, because the genome (RNA) of virus is $82 \%$ identical to the SARS coronavirus (SARS-CoV). As of April, there are about $25,00,000$ cumulative cases globally, with a $3.7 \%$ case-fatality rate. At present, no specific therapies for COVID-19 are available and research regarding the treatment of COVID-19 is infancy (Lu, 2020). Some preliminary studies have investigated potential combinations that include anti-malarial drug hydroxychloroquine, and anti-HIV vaccines can be used to treat COVID-19 infections. The main protease ( $\mathrm{M}^{\mathrm{pro}} /$ chymotrypsin-like protease also called as $3 \mathrm{CL}^{\mathrm{pro}}$ ) from COVID-19, represents a potential best target among coronaviruses (Rodríguez-Morales et al., 2020). The 3D structure of SARS-CoV-2 $\mathrm{M}^{\mathrm{pro}} / 3 \mathrm{CL}^{\mathrm{pro}}$ is highly identical to that of the SARS-CoV M $\mathrm{M}^{\mathrm{pro}} / 3 \mathrm{CL}^{\mathrm{pro}}$, as observed from the $96 \%$ sequence identity. Due to its key role on virus replication, Mpro is measured to be a key target for drug development to combat viral 
development. The $\mathrm{M}^{\mathrm{pro}} / 3 \mathrm{CL}^{\mathrm{pro}}$ protein may cleave polyprotein substrate at no less than 11 cleavage motifs containing Leu-Gln $\downarrow$ (Ser, Ala, Gly) sequences. Inhibiting the activity of $\mathrm{M}^{\mathrm{pro}} / 3 \mathrm{CL}^{\text {pro }}$ activity would block viral replication. Since in humans no proteases with similar cleavage specific are known, so inhibitors are unlikely to be toxic.

Traditionally, invention of new drug as therapeutic agents is a laborious and tedious task which may take about 12-14 years with lot of expenditure to bring a drug from discovery to market (Yang et al., 2018). Multidisciplinary approaches are routinely used in order to combat these challenges. A computer based in silico drug designing is a key platform in drug discovery which deliver a new drug in fast and cost-effective manner. Recently in the process of drug designing various bioinformatics tools helps in molecular docking analysis, protein/ligand interaction, virtual screening and in silico ADMET prediction.

Traditionally, aromatic plants are useful in the field of drug discovery as they are safer alternatives being utilized by humans for centuries (Sharma and Kaur, 2020). Previously, many of the new drug formulations are derived from natural products. Our present study focuses on the in-silico analysis of essential oil present in eucalyptus plants. Over the last many years, it has been used in various system of medicine. Its leaves, flowers, fruits and roots are used to treat various diseases like coughs, colds, asthma, skin infections, fever, bleeding piles and inflammation. Among several compounds, essential oils, from medicinal plants, have been reported to have antioxidant, antimicrobial and antiviral bioactivities, hence immensely used in cosmetic, pharmaceutical industries (Im et al., 2015). Essential oils are complex mixture of highly volatile substances like aldehydes, esters, terpenes, oxygenated derivatives and other aromatic compounds. In all the eucalyptus essential oils, pharmacologically, eucalyptol (1,8 cineole) is the major bioactive component (Zakaryan et al., 2017). Eucalyptus essential oil has been empirically used as antimicrobial agents, but little is known about its antiviral potential (Yang et al., 2017). We hypothesize that eucalyptol from Eucalyptus essential has the capability to prevent infection of COVID-19. Therefore, the research objective of the present study was in-silico analysis and comparative molecular docking studies pertain to eucalyptol in relation with Mpro protein. The present findings may provide other researchers with opportunity to identify the right drug to combat COVID-19. To further estimate the draggability of this phytochemical, the present study reports their ADMET profiles and potential biological activities using various in silico bioinformatics tools.

\section{Materials and Methods}

Proteins/macromolecules

COVID-19 $\mathrm{M}^{\mathrm{pro}} / 3 \mathrm{CL}^{\mathrm{pro}}$ structures were obtained from PDB (https://www.rcsb.org/).

\section{Ligand and drug scan}

Eucalyptol 3D structure was retrieved from PubChem (https://pubchem.ncbi.nlm.nih.gov/). Druglike properties (Lipinski's rule of five) and pharmacokinetic properties like ADMET (Absorption, Distribution, Metabolism, Excretion and Toxicity) were calculated using SWISSADME prediction (http://www.swissadme.ch/). Bioactivity analysis was carried out using molinspiration tool (https://www.molinspiration.com/cgi-bin/properties).

Binding mode of docked complexes

Eucalyptol and COVID-19 $\mathrm{M}^{\mathrm{pro}} / 3 \mathrm{CL}^{\mathrm{pro}}$ interaction were studied by using $\mathrm{pLip}$ webserver (https://projects.biotec.tu-dresden.de/plip-web/plip/index/) 
Determination of active sites

CASTp was used to find out the amino acids in the active site of COVID-19 $\mathrm{M}^{\mathrm{pro}} / 3 \mathrm{CL}^{\text {pro }}$ (http://sts.bioe.uic.edu/castp/index.html?2011).

\section{Molecular docking}

The docking study of the eucalyptol over COVID-19 $\mathrm{M}^{\mathrm{pro}} / 3 \mathrm{CL}^{\mathrm{pro}}$ was studied using 1-click docking (https://mcule.com/apps/1-click-docking/), PatchDock (https://bioinfo3d.cs.tau.ac.il/PatchDock/) and Swiss Dock (http://www.swissdock.ch/docking) software.

\section{Results and Discussion}

Traditionally, the discovery of new therapeutic drugs is a tedious and expensive process which generally takes 12-14 years with a lot of money to bring drug from discovery to market. In order to overcome these problems a lot many multidisciplinary approaches are used to discover new drug. In drug discovery processes, in silico drug designing is a form of computer-based modeling which is very useful. In the field of drug discovery, medicinal plants are advantageous as they are utilized as a safe herbal alternative by humans for centuries and many new drugs and active ingredients are derived from herbal products.

Being group of viruses, corona viruses (CoVs) mainly infect animals and humans. These viruses' effect respiratory, fever, cold, digestive, and liver system. In CoVs, it is the main protease $\left(\mathrm{M}^{\mathrm{pro}} / 3 \mathrm{CL}^{\mathrm{pro}}\right)$ found in the CoVs that play essential roles in viral replication and associated with the severe acute respiratory syndrome (SARS), which can be accessed in PDB and was suggested to be a potential drug target for 2019$\mathrm{nCov}(\mathrm{Lu}, 2020)$ hence often used as protein targets during the development of antiviral therapeutics. $\mathrm{M}^{\mathrm{pro}} / 3 \mathrm{CL}^{\mathrm{pro}}$ protease structure invention in COVID-19 provides a nice path to identify potential drug candidates to prevent infection. As cited by Liu and Wang (2020) protein sequences of the SARS-CoV $\mathrm{M}^{\mathrm{pro}} / 3 \mathrm{CL}^{\mathrm{pro}}$ and the $2019-\mathrm{nCoV} \mathrm{M}^{\mathrm{pro}} / 3 \mathrm{CL}^{\mathrm{pro}}$ are $96 \%$ identical so can be used as potential therapeutic targets.

In the present study, we performed in silico analysis of eucalyptus essential oil component eucalyptol (1-8 cineole) against $\mathrm{M}^{\mathrm{pro}} / 3 \mathrm{CL}^{\text {pro }}$ protein of COVID-19. Molecular docking is a widely used computational tool in modern drug discovery process. It predicts the binding affinity of ligands with the target receptor protein. The efficacy of the docked complex was evaluated on the basis of two essential criteria's: The minimum binding energy and the interaction of the ligand with the active site residues. A ligand undergoes either hydrogen bonding or hydrophobic interactions or both while docking into the active site. The results of docking can be used to find the best inhibitors for specific target proteins and thus to design new drugs. The ligand (eucalyptol) was docked to $\mathrm{M}^{\mathrm{pro}} / 3 \mathrm{CL}^{\text {pro }}$ using 1-click and patch Dock and Swiss Dock softwares. Docking interactions showing full fitness and Gibbs free energy prediction was revealed by Swiss Dock.

Molecular docking of eucalyptol ( 1,8 cineole) with $\mathrm{M}^{\text {pro }} / 3 \mathrm{CL}^{\text {pro }}$ protein revealed 4 different poses. Highest negative values indicated maximum binding affinity of the pose. Docking pose and molecular interactions of eucalyptol are shown in Figures 1 and 2. Eucalyptol was successfully docked with $\mathrm{M}^{\mathrm{pro}} / 3 \mathrm{CL}^{\mathrm{pro}}$ protein binding pocket in domains I and II with good scores (Figure 1, Table 1). The eucalyptol showed full fitness of- $2291.07 \mathrm{kcal} / \mathrm{mol}$ and estimated $\Delta \mathrm{G}$ of $-6.04 \mathrm{kcal} / \mathrm{mol}$ within active site amino acids of $\mathrm{M}^{\mathrm{pro}} / 3 \mathrm{CL}^{\mathrm{pro}}$ protein of COVID-19. The interaction of Eucalyptol in the binding pocket of $\mathrm{M}^{\mathrm{pro}} / 3 \mathrm{CL}^{\mathrm{pro}}$ was mediated by two hydrophobic interactions via MET6 at atomic distances of 3.42, PHE8 at atomic distances

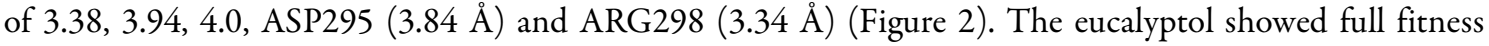
within active site amino acids of $\mathrm{M}^{\mathrm{pro}} / 3 \mathrm{CL}^{\text {pro }}$ protein of COVID-19 (Figure 1). CAST-P server also revealed the presence of interactive residues as active site residue of cavities in $\mathrm{M}^{\text {pro }} / 3 \mathrm{CL}^{\text {pro }}$ protein (Figure 3 , Table $S 1$ ). Domains I and II, representing residues 10-99 and 100-182, respectively, are six-stranded antiparallel $\beta$ barrels that harbor the substrate-binding site between them. A globular cluster of five helices, domain III (residues 198-303), is involved in regulating dimerization of the Mpro, mainly by a salt-bridge interaction 
between both protomers (Zhang et al., 2020). The dimer formed by $\mathrm{M}^{\mathrm{pro}} / 3 \mathrm{CL}^{\text {pro }}$ protein of COVID-19 has a contact interface, mainly between domain II of protomer A and the $\mathrm{NH}$-terminal residues (N-finger") of protomer $\mathrm{B}$, with the two molecules oriented perpendicular to one another (Figure 1). It was cited that for $\mathrm{M}^{\mathrm{pro}} / 3 \mathrm{CL}^{\mathrm{pro}}$ enzyme catalytic activity, dimerization is essential, because the $\mathrm{N}$-finger of each of the two protomers interacts and thereby helps shape the $S 1$ pocket of the substrate-binding site (Tan et al., 2005). To reach this interaction site, the $\mathrm{N}$-finger is squeezed in between domains II and III of the parent monomer and domain II of the other monomer. Antiviral bioactivities of many compounds like flavonoids, terpenoids and phenolics from essential oils, have been reported (Goodger et al., 2016; Zakaryan et al., 2017; Yang et al., 2018). The present study investigated eucalyptol (1,8 cineole) as key inhibitor of the $\mathrm{M}^{\mathrm{pro}} / 3 \mathrm{CL}^{\mathrm{pro}}$. Hydroxy groups $(-\mathrm{OH})$, ketone groups $(=\mathrm{O})$ and ether groups (-O-) in eucalyptol compounds are predicted to play roles amino acid residue interactions at the active site of COVID-19 Mpro. RMSD data indicated good affinity of ligand with protein structure with RMSD values.

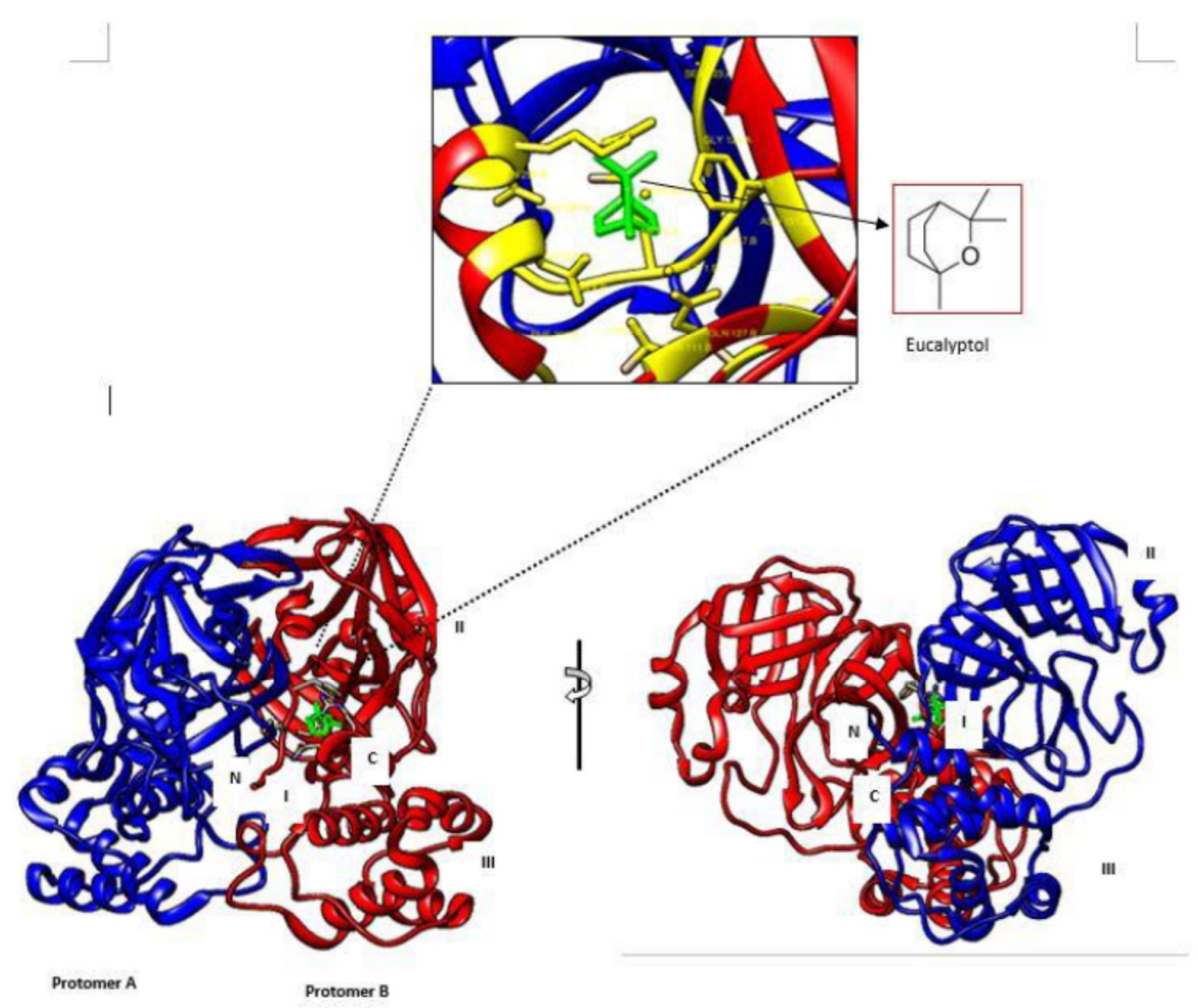

Figure 1. Three-dimensional docked structure of SARS-CoV-2 Mpro with eucalyptol, in two different views.

One protomer of the dimer is shown in light blue, the other one in red. Domains are labeled by Roman numbers. Chain termini are labeled N and C for Protomer A (blue) and for Protomer B (red). 
Table 1. Binding Energy and full fitness values of Docked complex and interaction values

\begin{tabular}{|c|c|c|c|c|c|}
\hline \multirow{2}{*}{ Tool used } & \multirow{2}{*}{ Dock pose } & \multirow{2}{*}{ Dock score } & \multicolumn{2}{|c|}{$\begin{array}{c}\text { Interacting residues and } \\
\text { length }\left(4 \mathrm{~A}^{\circ}\right)\end{array}$} & \multirow{2}{*}{$\begin{array}{l}\text { Binding pocket residues } \\
\text { within } 4 \mathrm{~A}^{\circ} \text { radius }\end{array}$} \\
\hline & & & $\begin{array}{c}\text { H-bond } \\
\text { interactions }\end{array}$ & $\begin{array}{l}\text { Hydrophobic } \\
\text { interactions }\end{array}$ & \\
\hline 1-click & 1 & -5.2 & \multirow{6}{*}{ - } & \multirow{6}{*}{$\begin{array}{c}\text { MET6, } \\
\text { PHE6,8, } \\
\text { ASP295, ARG } \\
298\end{array}$} & \multirow{6}{*}{$\begin{array}{l}\text { PHE6,8, ARG4, MET6, } \\
\text { SER123, GLY124, } \\
\text { ASN151, ALA7, } \\
\text { GLN127, ARG298, } \\
\text { PHE291, 294, ASP295, } \\
\text { GLN299 }\end{array}$} \\
\hline \multirow[t]{2}{*}{ PatchDock } & Score & ACE & & & \\
\hline & 3122 & -60.19 & & & \\
\hline \multirow[t]{3}{*}{ SwissDock } & Full fitness & Estimated $\Delta \mathrm{G}$ & & & \\
\hline & & & & & \\
\hline & 2291.07 & 6.04 & & & \\
\hline
\end{tabular}
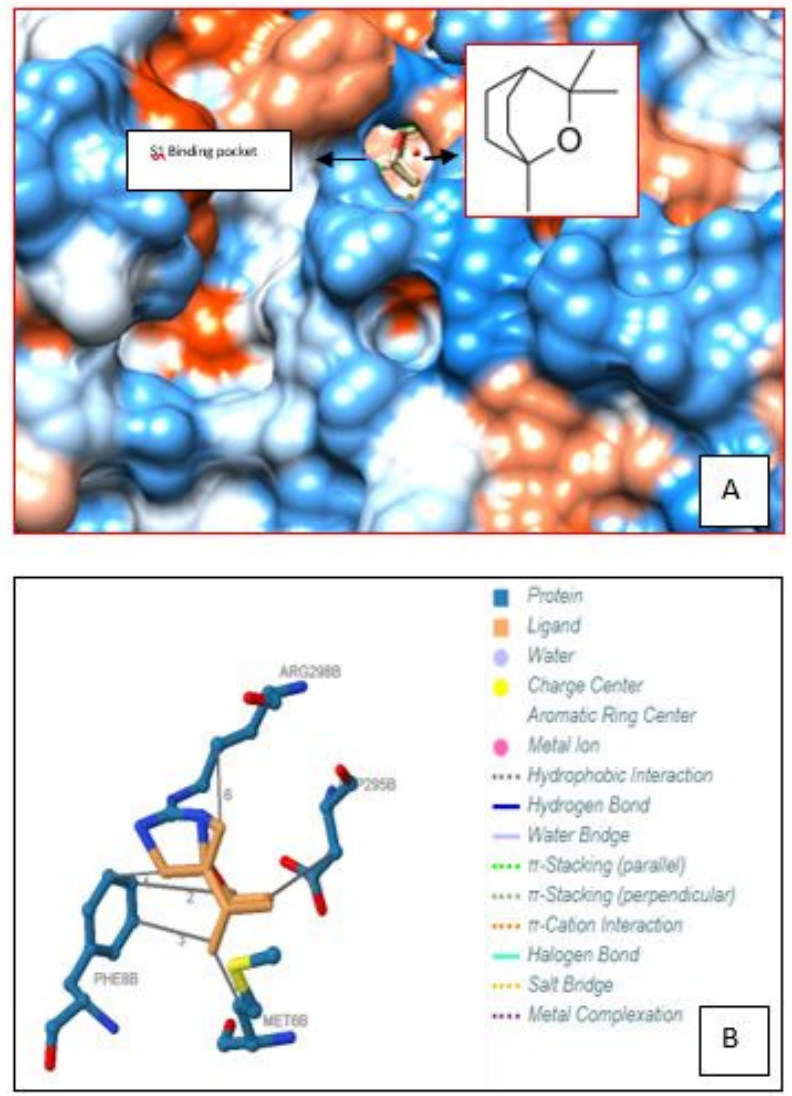

Figure 2. Surface docked 3-D structure of SARS-CoV-2 Mpro (A), and molecular interactions (B) of eucalyptol with Mpro protein 


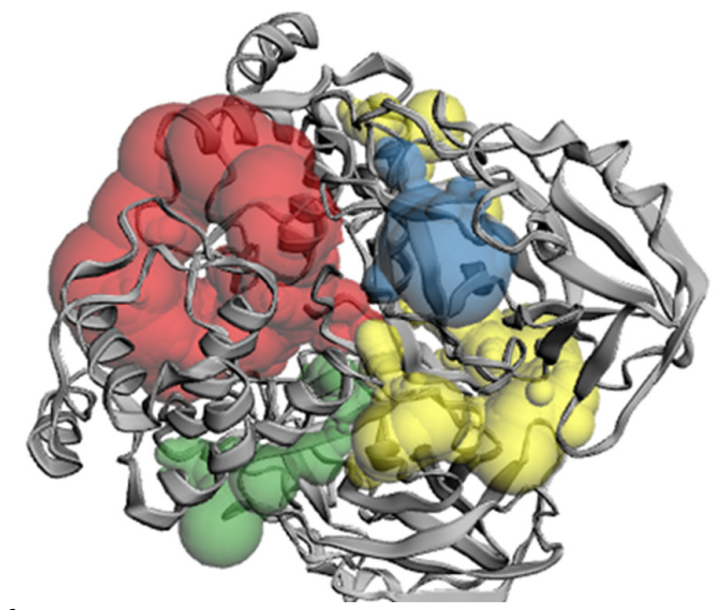

Figure 3. Active sites of Mpro protein

\begin{tabular}{|c|c|c|c|c|}
\hline Pocket ID & Area (SA) & Volume (SA) & $\begin{array}{c}\text { Negative volume } \\
\text { colour }\end{array}$ & Actives residues \\
\cline { 1 - 4 } 1 & 1689.804 & 2332.004 & Red & \multirow{2}{*}{$\begin{array}{c}\text { Supplementary } \\
\text { Table 1 }\end{array}$} \\
\hline 2 & 1470.951 & 1288.702 & Yellow & Blue \\
\hline 3 & 266.905 & 290.390 & Green & \\
\hline 4
\end{tabular}

Contact plot analysis indicated the role of interactions between Mpro and eucalyptol (Figure 4). The comparison of $\mathrm{HB}$ type contact plots of open (without ligand) and closed (with ligand) structures of $\mathrm{M}^{\text {pro }} / 3 \mathrm{CL}^{\text {pro }}$ revealed no large-scale conformational rearrangements between two states as indicated. However, side chain and residue type contact plots indicated significant conformational rearrangements via side chain and hydrophobic interactions upon eucalyptol ligand binding to $\mathrm{M}^{\text {pro }} / 3 \mathrm{CL}^{\text {pro }}$ protein of SARS-CoV-2. This suggests high structural stability and may lead to high inhibitory activity of eucalyptol on $\mathrm{M}^{\text {pro }} / 3 \mathrm{CL}^{\text {pro }}$ active site.

Pharmacokinetic analysis using ADMET properties was studied. Eucalyptol ligand used in this study did not violate Lipinski's rule of five (Lipinski, 2004, Shukla et al., 2014). Eucalyptol scanning results are illustrated in Table 2. Topological polar surface area (TPSA) value was considerably less than $90^{\circ} \mathrm{A}$ squared. It indicated good permeability of eucalyptol cell membranes to enter Blood Brain Barrier (BBB). Pharmacokinetics parameters and ADMET factors are key parameters for success of mostly drugs during clinical trials. Blood Brain Barrier (Log BB) permeability which was good with eucalyptol. The eucalyptol showed positive results for $\mathrm{BBB}$ profile. Log $\mathrm{Po} / \mathrm{w}$ (lipophilicity indicator, octanol-water partition coefficient) was 2.67, indicating eucalyptol was optimal BBB penetration. Eucalyptol did not show any affinity with P-glycoprotein (p-gp), a efflux transporter located at BBB. GI (Gastrointestinal tract absorption) of eucalyptol was high (Table 2). In order to exert a toxic effect, drug molecules have to be absorbed from intestinal tract in the body. Further, eucalyptol shown non inhibitory activity against cytochrome P series (CYP1-3) of enzymes, involved in liver detoxification of toxins from body. 3D molecular structures showing Molecular Lipophilicity Potential (MLP) and Polar Surface Area (PSA) are also shown in Fig. 5. MLP is convenient property to rationalize numerous molecular ADME characteristics (for example: plasma-protein binding or membrane penetration). Analysis of 3D distribution of hydrophobicity on molecular surface is predominantly helpful when explaining differences is observed in ADME properties of molecules with the same logP. Bioactivity of eucalyptol as the drug was calculated online by using Molinspiration drug-likeness score. Table 2 depicts Bioactivity score of eucalyptol. Ion channel property of Eucalyptol was high $(>0)$ while enzyme inhibitor and protein inhibitor activities were moderate $(<0)$. 

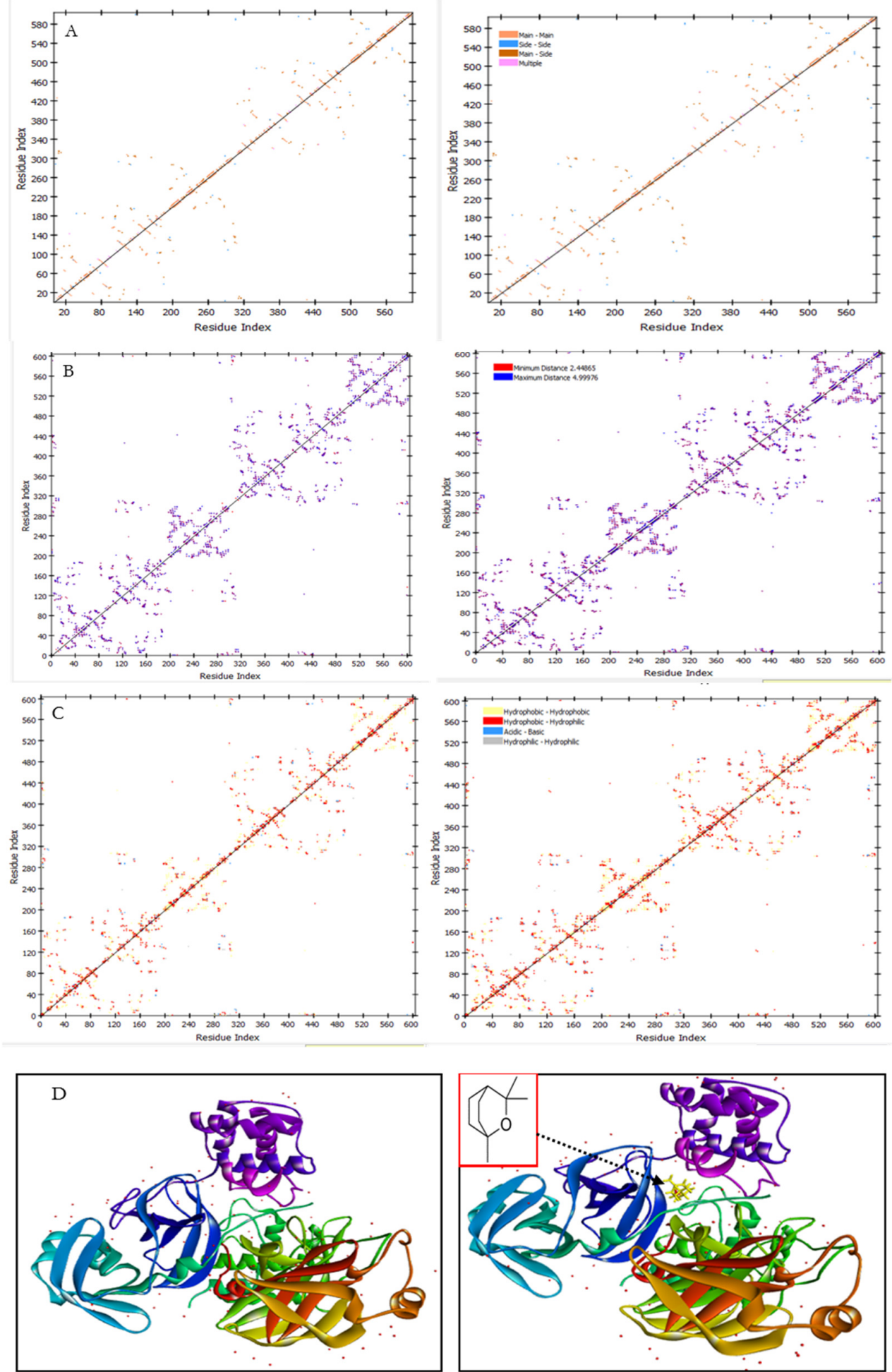

Mpro

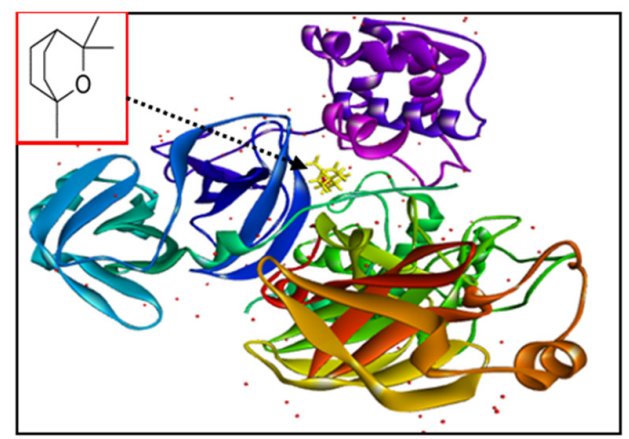

Mpro-ligand complex

Figure 4. Contact plot analysis of Mpro and Mpro-eucalyptol complexes. A: HB type plot; B: Side chain Type plot; C: Residue Type plot; D: Mpro and Mpro-eucalyptol structures. Arrows indicate major changes 
Table 2. Pharmacokinetic properties $\mathrm{M}^{\mathrm{pro}} / 3 \mathrm{CL}^{\text {pro }}$ potential inhibitor eucalyptol

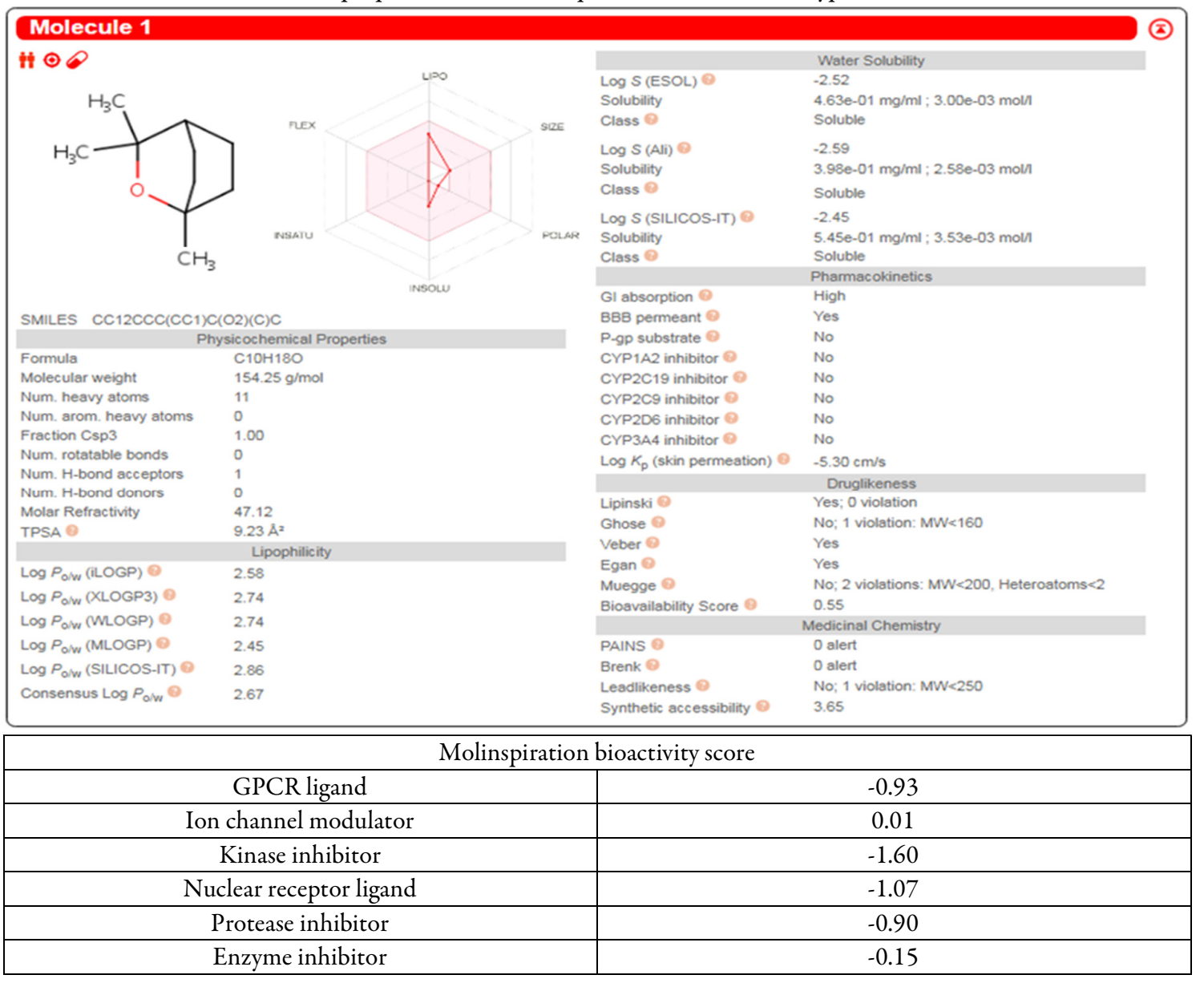

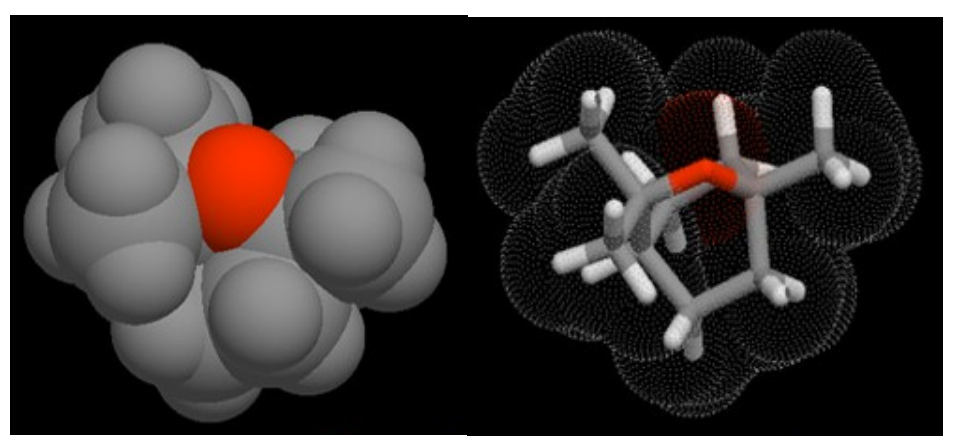

Figure 5. Molecular lipophilicity potential (MLP)/ polar surface area (PSA) views of eucalyptol. Hydrophobic areas: encoded by violet; Hydrophilic areas: red

\section{Conclusions}

SARS-Cov-2 has emerged as major pandemic worldwide. Present studs revealed molecular docking of eucayptol (1,8 cineole) from eucalyptus essential against COVID-19 S protein. This study suggested that 
Eucalyptol has the potential to act as potential inhibitors of SARS-Cov-2- $\mathrm{M}^{\mathrm{pro}} / 3 \mathrm{CL}^{\mathrm{pro}}$ protein. However, more in vivo and in vitro model based studies may pave way these compounds in drug discovery.

\section{Authors' Contributions}

ADS: designed the study and prepared manuscript. IJK: designed the study and prepared manuscript. Both authors read and approved the final manuscript.

\section{Ethical approval}

This article does not contain any studies involving animals or human participants performed by any of the authors.

\section{Acknowledgements}

ADS want to thank management for support.

\section{Conflict of Interests}

The authors declare that there are no conflicts of interest related to this article.

\section{References}

Goodger JQD, Seneratne SL, Nicolle D, Woodrow IE (2016). Foliar essential oil glands of eucalyptus subgenus Eucalyptus (Myrtaceae) are a rich source of flavonoids and related non-volatile constituents. PLoS ONE 11(3):e0151432. https://doi.org/10.1371/journal.pone.0151432

Im K, Kim J, Min H (2015). Ginseng, the natural effectual antiviral: Protective effects of Korean red ginseng against viral infection. Journal of Ginseng Research 40(4):309-314. https://doi.org/10.1016/j.jgr.2015.09.002

Lipinski CA (2004). Lead- and drug-like compounds: the rule-of-five revolution. Drug Discovery Today: Technologies 1:337-341.https://doi.org/10.1016/j.ddtec.2004.11.007

Liu X, Wang XJ (2020). Potential inhibitors against 2019-nCoV coronavirus M protease from clinically 13 of 14 approved medicine. Journal of Genetics and Genomics 47(2):119-121. https://doi.org/10.1016/j.jgg.2020.02.001.

Lu H (2020). Drug treatment options for the 2019-new coronavirus (2019-nCoV). Bioscience Trends 14(1):69-71. https://doi.org/10.5582/bst.2020.01020

Rodríguez-Morales P, Alfonso J, MacGregor K, Kanagarajah S, Dipti P (2020). Going global - travel and the 2019 novel coronavirus. Travel Medicine and Infectious Disease 33:101578. https://doi.org/10.1016/j.tmaid.2020.101578

Tan J, Verschueren KHG, Anand K, Shen J, Yang M, Xu Y, ... Hilgenfeld R (2005). pH-dependent conformational flexibility of the SARS-CoV main proteinase (Mpro) dimer: Molecular dynamics simulations and multiple X-ray structure analyses. Journal of Molecular Biology 354(1):25-40. https://doi.org/10.1016/j.jmb.2005.09.012

Sharma AD, Kaur I (2020). Molecular docking studies on jensenone from eucalyptus essential oil as a potential inhibitor of COVID 19 corona virus infection. Research and Reviews in Biotechnology and Biosciences 1:59-66.

Shukla A, Sharma P, Prakash O, Singh M, Kalani K, Khan F, ... Srivastava SK (2014). QSAR and docking studies on Capsazepine derivatives for immunomodulatory and anti- inflammatory activity. PLoS One 9(7):e100797. https://doi.org/10.1371/journal.pone.0100797 
Yang L, Wen KS, Ruan X, Zhao YX, Wei F, Wang Q (2018). Response of plant secondary metabolites to environmental factors. Molecules 23:1-26. https://doi.org/10.3390/molecules23040762

Zakaryan H, Arabyan E, Oo A, Zandi K (2017). Flavonoids: promising natural compounds against viral infections. Archives of Virology 162:2539-2551. https://doi.org/10.1007/s00705-017-3417-y

Zhang L, Lin D, Sun X, Curth U, Drosten C, Sauerhering L, ... Hilgenfeld R (2020) Crystal structure of SARS-CoV-2 main protease provides a basis for design of improved -ketoamide inhibitors. Science 368(6489):409-412. https://doi.org/10.1126/science.abb3405

OPEN ACCESS

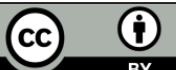

The journal offers free, immediate, and unrestricted access to peer-reviewed research and scholarly work. Users are allowed to read, download, copy, distribute, print, search, or link to the full texts of the articles, or use them for any other lawful purpose, without asking prior permission from the publisher or the author.

License -Articles published in Notulae Scientia Biologicaeare Open-Access, distributed under the terms and conditions of the Creative Commons Attribution (CC BY 4.0) License.

(c) Articles by the authors; SHST, Cluj-Napoca, Romania. The journal allows the author(s) to hold the copyright/to retain publishing rights without restriction. 